\title{
Sensitive Method for Biomolecule Detection Utilizing Signal Amplification with Porphyrin Nanoparticles
}

Supporting Information

Lauren E. Gibson, David W. Wright*

Department of Chemistry, Vanderbilt University, Station B 351822, Nashville, TN 37235-1822, USA

Fax: 615-343-1234; Tel: 615-322-2636

*David.Wright@Vanderbilt.edu

Table of Contents

Molar extinction coefficient of tetra(4-carboxyphenyl)porphyrin..................S-2

Size stability of tetra(4-carboxyphenyl)porphyrin nanoparticles...................3

$\mathrm{pH}$ stability of tetra(4-carboxyphenyl)porphyrin nanoparticles................... -4

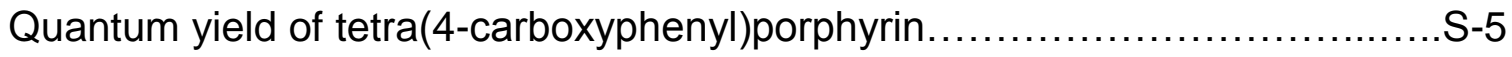

Fluorescence of tetra(4-carboxyphenyl)porphyrin nanoparticles..................6

Fluorescent stability of tetra(4-carboxyphenyl)porphyrin.......................

Reaction mechanism for diazirine photoreactive linker........................ -8

Specificity of Ab-conjugated tetra(4-carboxyphenyl)porphyrin nanoparticles....S-9

Optimization of TCPP NP assay for detection of Rab-lgG.................. $-10,11$

Optimization of TCPP NP assay for detection of pfHRPII................S-12,13,14 


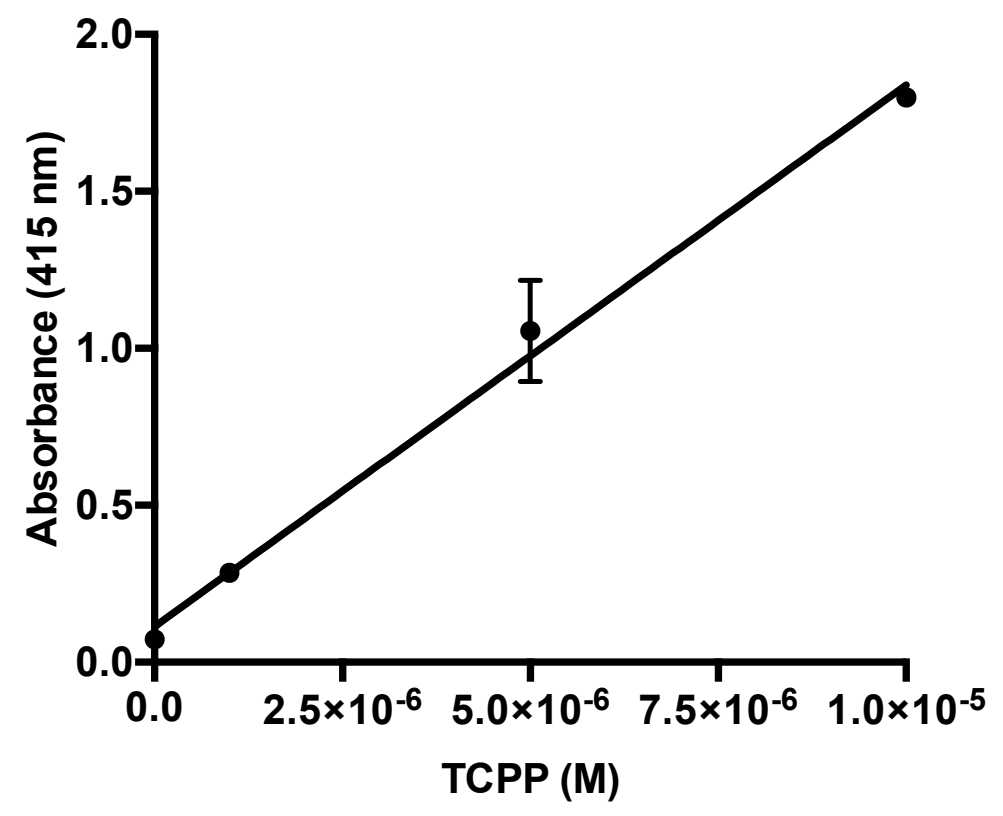

Figure S-1. Standard curve used to determine the molar extinction coefficient of TCPP in $0.1 \mathrm{M} \mathrm{NaOH} . \mathrm{y}=172656 \mathrm{x}-6.52 \times 10^{-7}, \mathrm{R}^{2}=0.986$. 


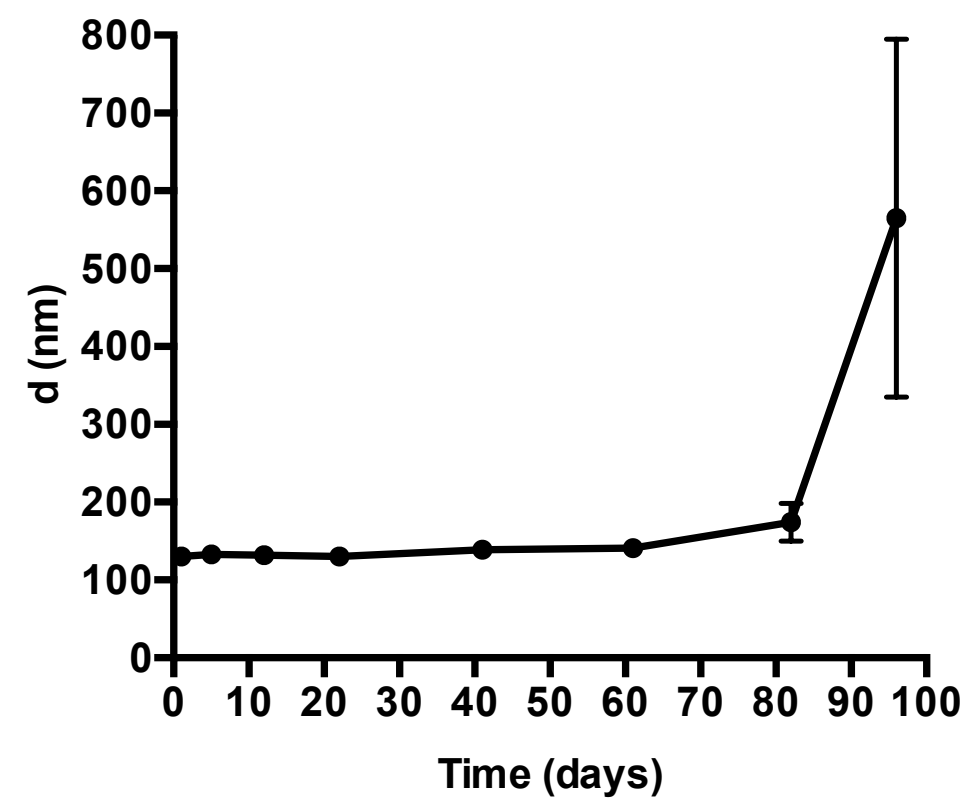

Figure S-2. The increase in diameter of the TCPP NPs over time. Aggregation of the TCPP NPs is observed after 90 days. 


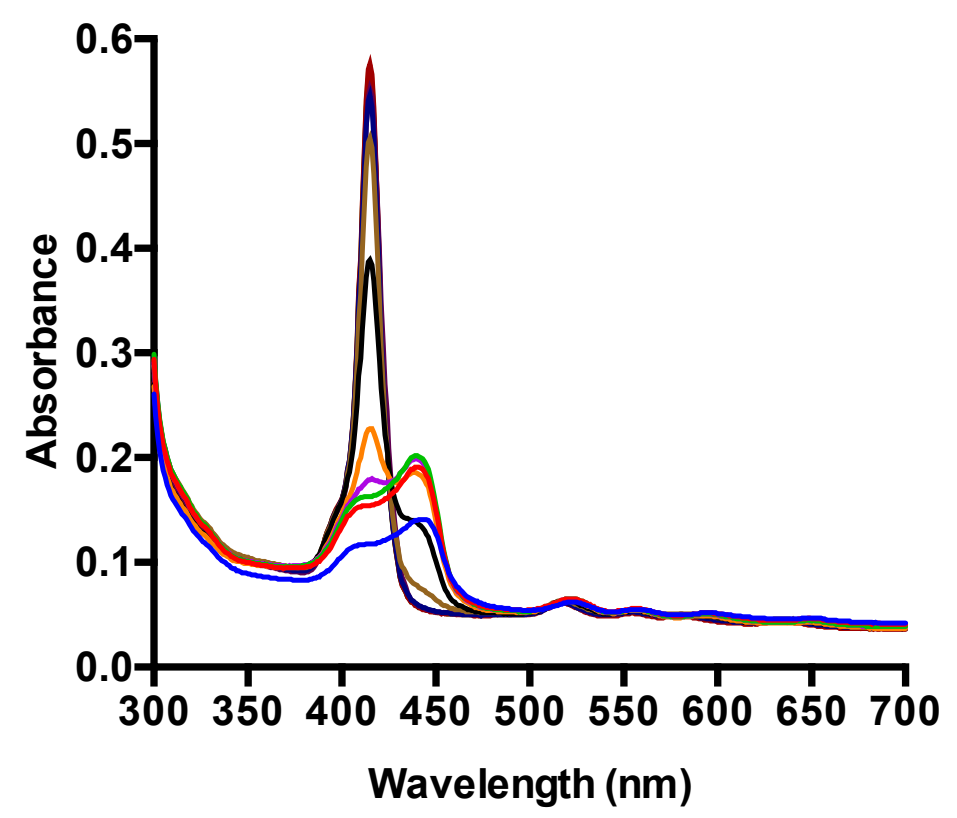

$-\mathrm{pH} 3$

$-\mathrm{pH} 4$

$-\mathrm{pH} 5$

$-\mathrm{pH} 6$

- $\mathrm{pH} 6.5$

$-\mathrm{pH} 6.8$

$-\mathrm{pH} 7$

- $\mathrm{pH} 8$

$-\mathrm{pH} 9$

$-\mathrm{pH} 10$

- $\mathrm{pH} 11$

$\mathrm{pH} 12$

Figure S-3. The absorbance spectrum of TCPP NPs as a function of $\mathrm{pH}$. The increase in the Soret band at $415 \mathrm{~nm}$, with increasing $\mathrm{pH}$, can be seen. 


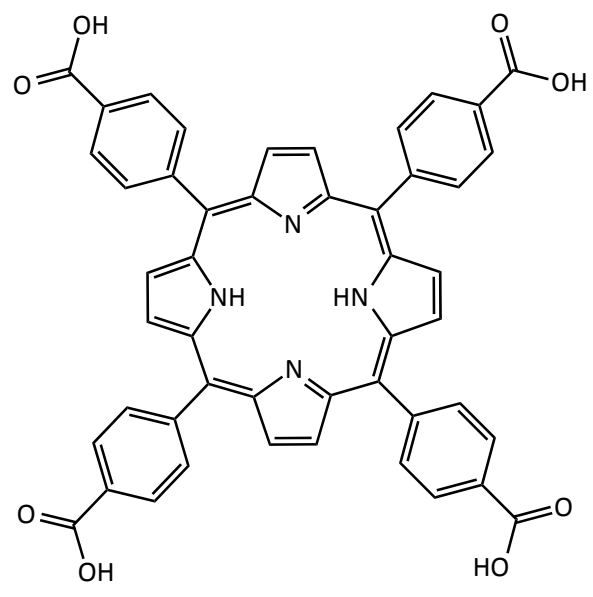

Sample $=$ Tetra(4-carboxyphenyl)porphyrin $\quad$ Reference $=$ Tetraphenylporphyrin

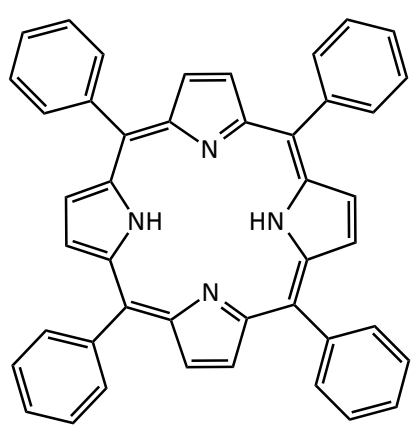

The quantum yield of tetra(4-carboxyphenyl) porphyrin (TCPP) in $0.1 \mathrm{M}$ sodium hydroxide $(\mathrm{NaOH})$ was determined using tetraphenylporphyrin $\left(\mathrm{H}_{2} \mathrm{TPP}\right)$ as a reference. The quantum yield $(\phi)$ was calculated according to the following equation:

$$
\phi_{s}=\phi_{r}\left(\frac{n_{s}^{2} A_{r} I_{s}}{n_{r}^{2} A_{s} I_{r}}\right)
$$

where $s=$ sample, $r$ = reference, $n=$ refractive index of the solvent, $A=$ absorbance, and $\mathrm{I}=$ intensity (fluorescence peak area). According to literature the quantum yield of $\mathrm{H}_{2}$ TPP is 0.13 in methanol. ${ }^{1}$ The refractive index of methanol is 1.3284 at $20^{\circ} \mathrm{C}$ and that of $0.1 \mathrm{M} \mathrm{NaOH}$ is $1.33696 .^{2}$ A solution of each porphyrin was made with the same absorbance at $415 \mathrm{~nm}$, the fluorescence spectrum of these solutions was taken and the areas of the peaks calculated. Using these values the quantum yield of TCPP in $0.1 \mathrm{M} \mathrm{NaOH}$ was found to be 0.231 . 


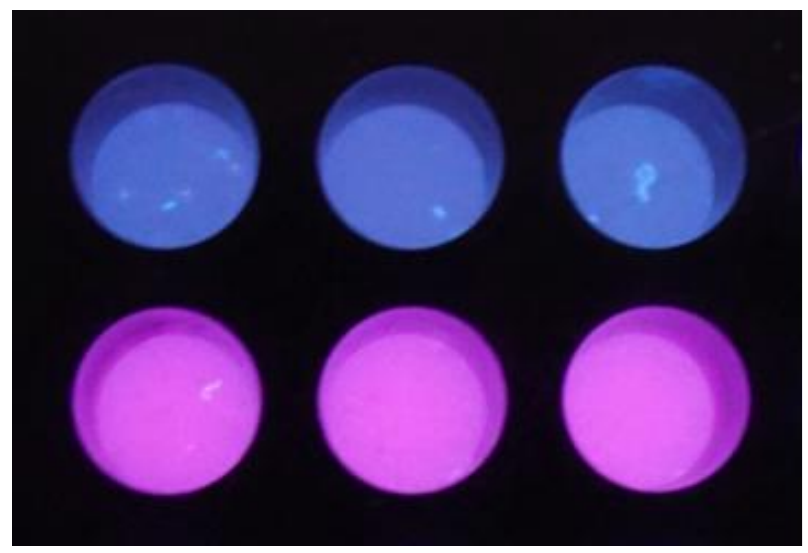

Before NP Dissolution

\author{
After NP Dissolution
}

Figure S-4. TCPP NP fluorescence (top) and TCPP fluorescence after dissolution of the TCPP NPs with base (bottom). It can be seen that fluorescence is quenched in the nanoparticle form but is very strong after the TCPP is released from the nanoparticle with dissolution by sodium hydroxide. Picture taken on UV transilluminator, excitation wavelength $365 \mathrm{~nm}$. Maximum fluorescence would be seen with an excitation wavelength of $415 \mathrm{~nm}$. 


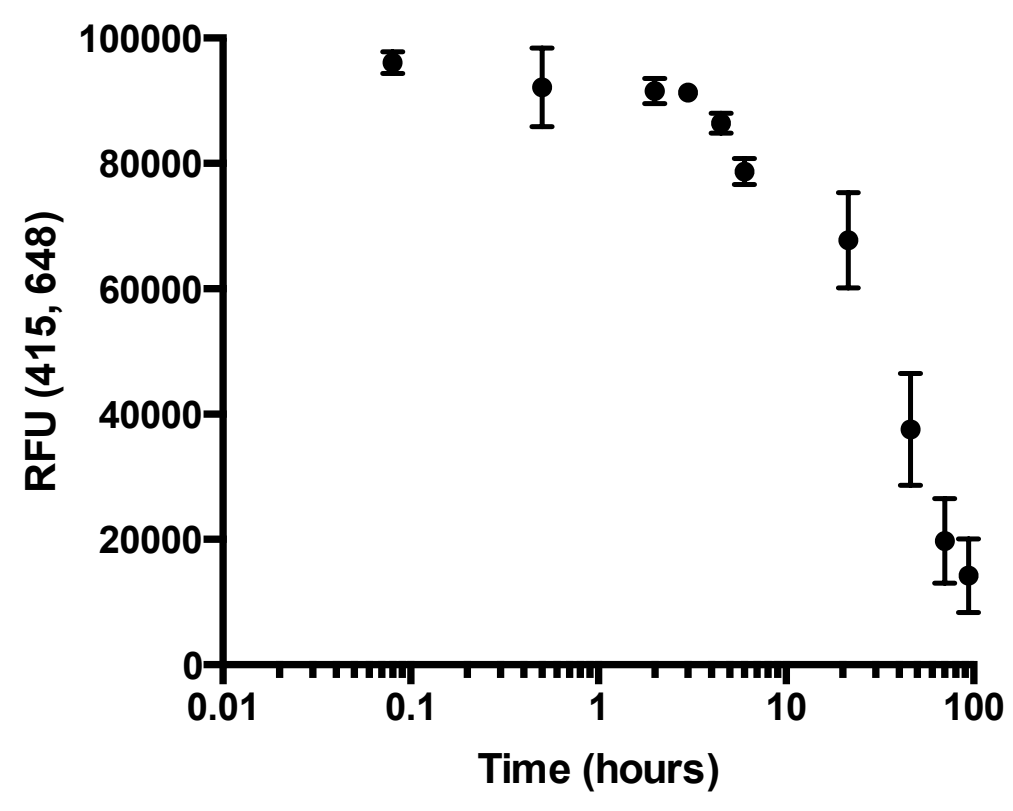

Figure S-5. Degradation of the fluorescent signal of TCPP molecules in $0.1 \mathrm{M}$ $\mathrm{NaOH}$ after TCPP NP dissolution. The solution was left on the benchtop, exposed to white light, for the duration of the experiment. The fluorescent signal did not decrease for 4.5 hours. 

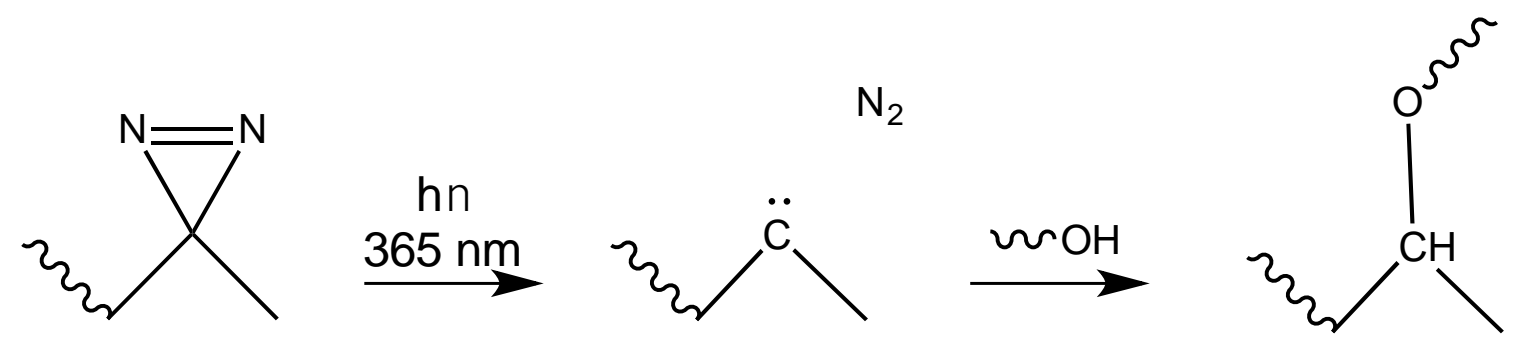

Figure S-6. Reaction of the diazirine group with nucleophilic $\mathrm{OH}$ groups in the presence of UV light. The TEGME and TCPP components of the TCPP NPs contain $\mathrm{OH}$ groups, so these groups are a probable location of the covalent bond between the TCPP NPs and antibodies modified with the diazirine photoreactive linker. 


\begin{tabular}{|c|c|c|}
\hline Detection Element & Rab-IgG (nM) & Average RFU (415, 648) \\
\hline Ab-TCPP NP & $0 \mathrm{nM}$ & $940 \pm 60$ \\
\hline TCPP NP & $20 \mathrm{nM}$ & $433 \pm 11$ \\
\hline Ab-TCPP NP & $20 \mathrm{nM}$ & $84000 \pm 3000$ \\
\hline
\end{tabular}

Table S-1. Table contrasting the signal of the TCPP NP assay for detection of Rab-lgG when Ab-TCPP NPs are used as opposed to unmodified TCPP NPs. It can be seen that signal for Rab-lgG is specific. 


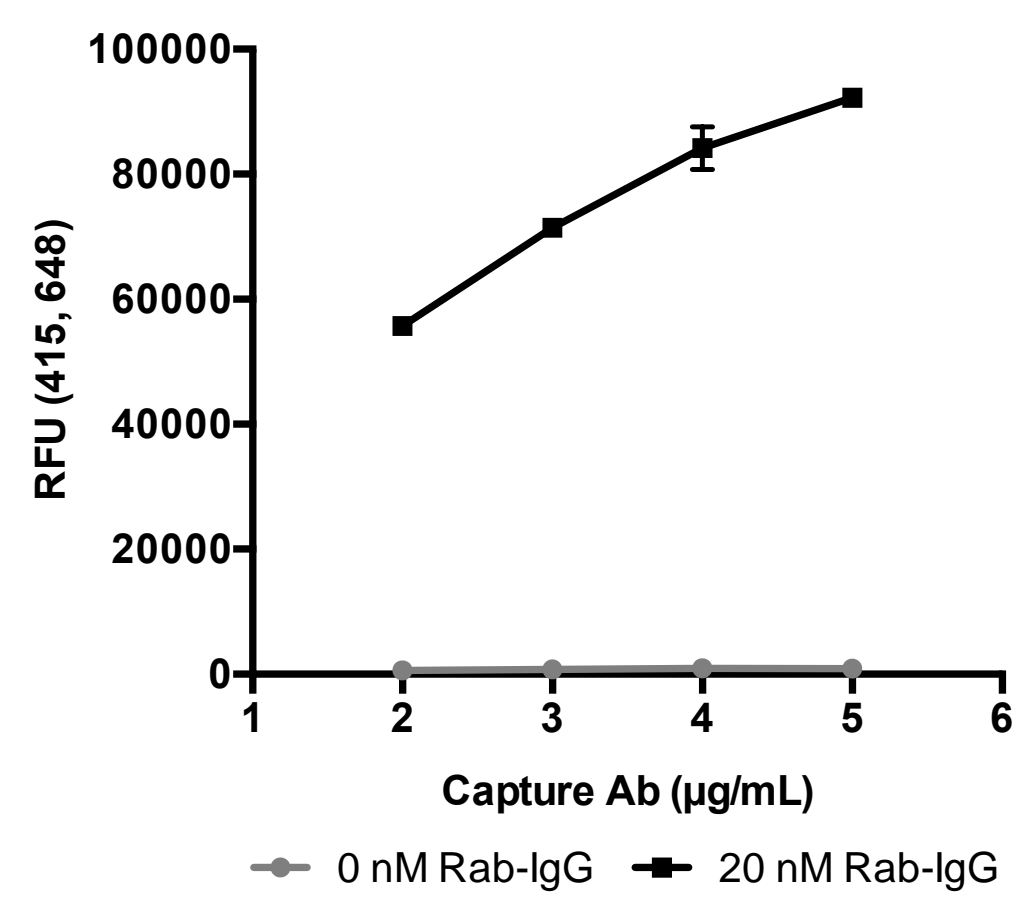

Figure S-7. Optimization of the capture antibody concentration for the sandwich Rab-IgG assay. The signal increases with greater amounts of capture antibody. It can be seen that the signal begins to level off at $4 \mu \mathrm{g} / \mathrm{mL}$ of capture antibody and that the background signal ( $0 \mathrm{nM}$ Rab-lgG) remains lnw. 


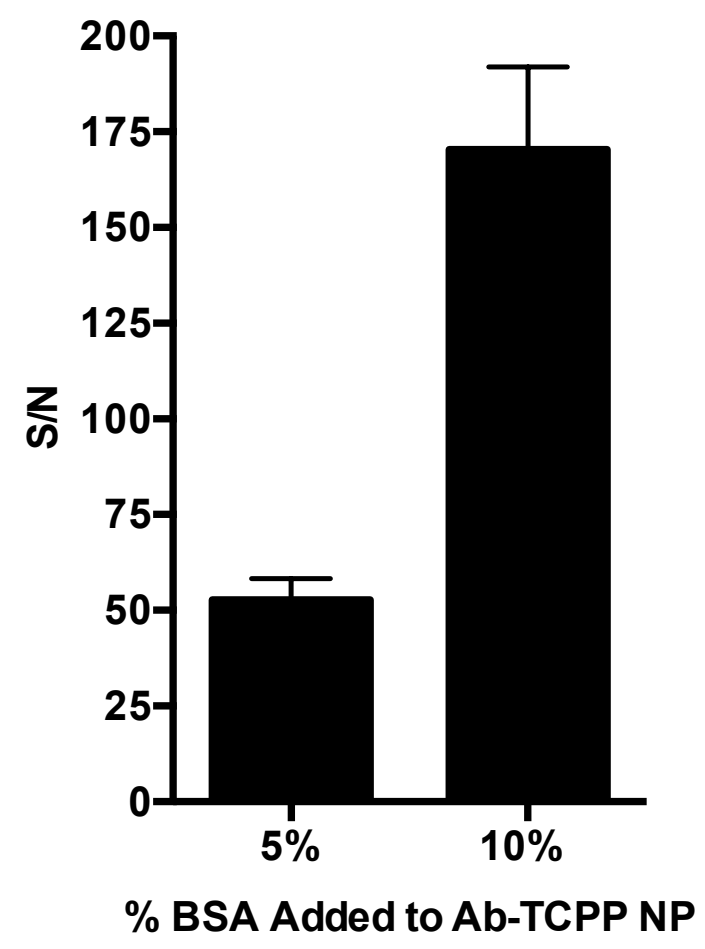

Figure S-8. Blocking of Ab-TCPP NPs with BSA. Increasing BSA concentration increases signal to noise of the assay for detection of Rab-lgG. 


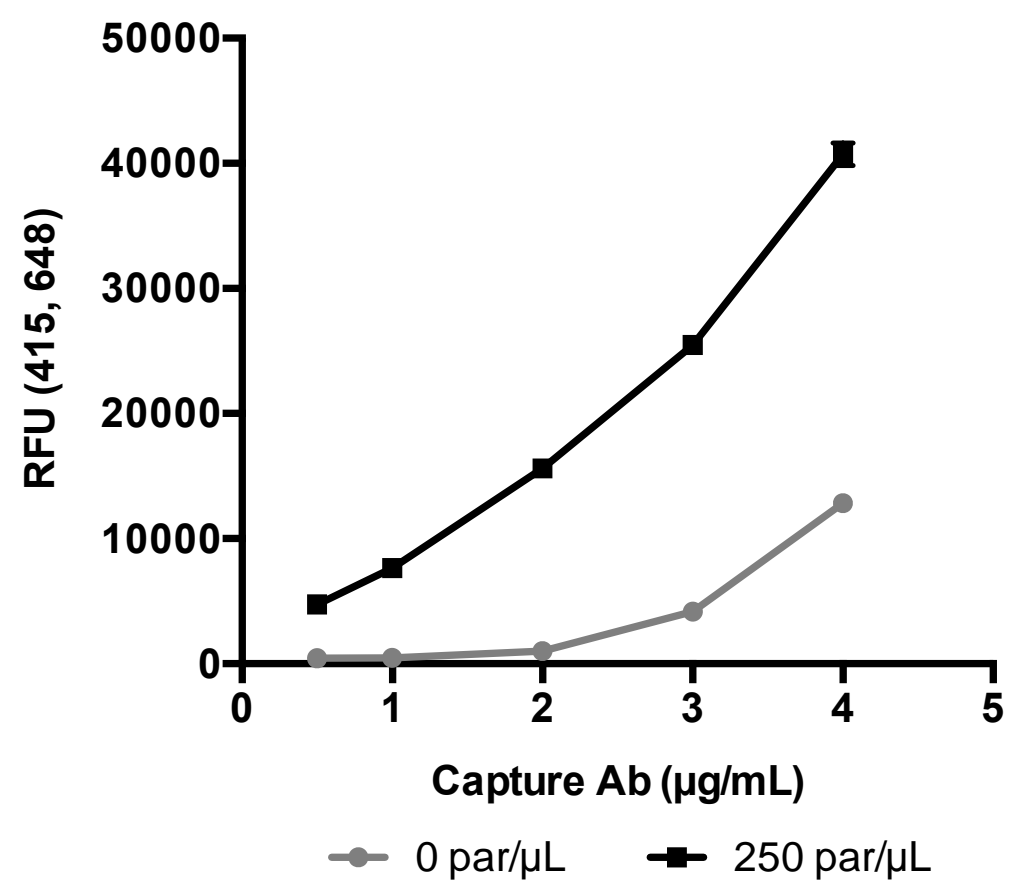

Figure S-9. Optimization of the capture antibody concentration for the sandwich pfHRPII assay. The signal increases with greater amounts of capture antibody. It can be seen that at $3 \mu \mathrm{g} / \mathrm{mL}$ of capture antibody the background signal ( 0 par $/ \mu \mathrm{L}$ ) substantially increases. Thus, $2 \mu \mathrm{g} / \mathrm{mL}$ of capture antibody was chosen for maximum signal to noise performance. 


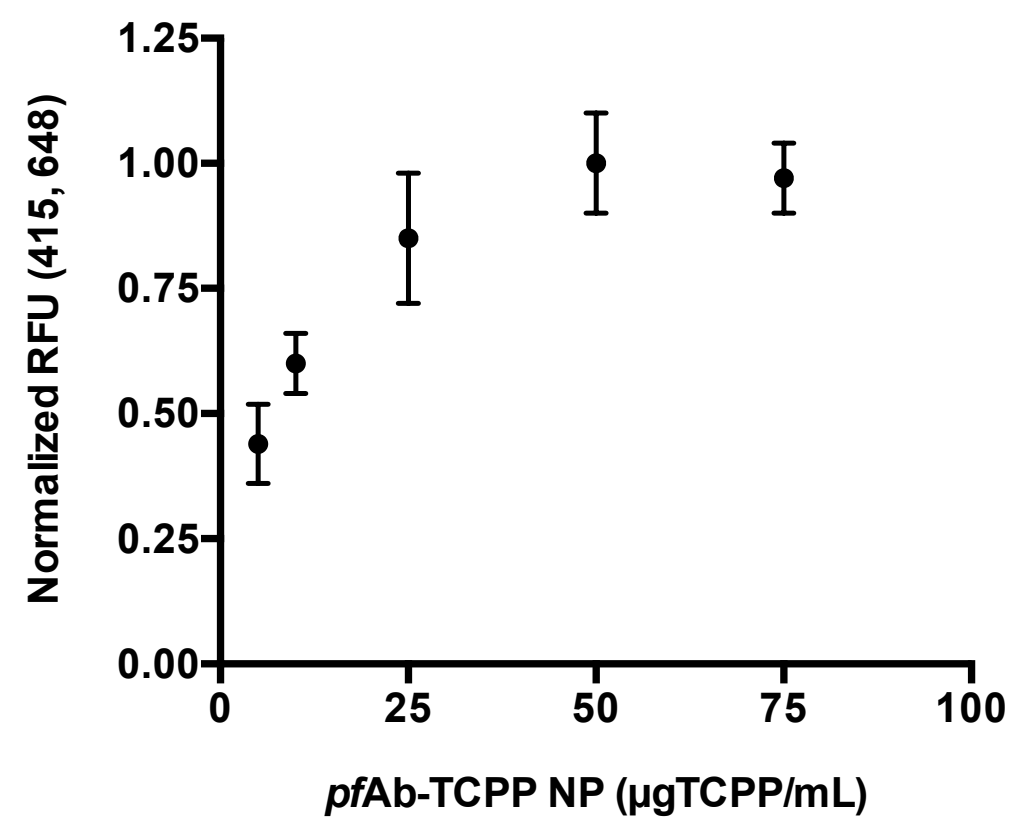

Figure S-10. Normalized signal at various pfAb-TCPP NP concentrations with constant pfHRPII (250 par/ $\mu \mathrm{L})$. The signal saturates at $50 \mu \mathrm{gTCPP} / \mathrm{mL}$. 


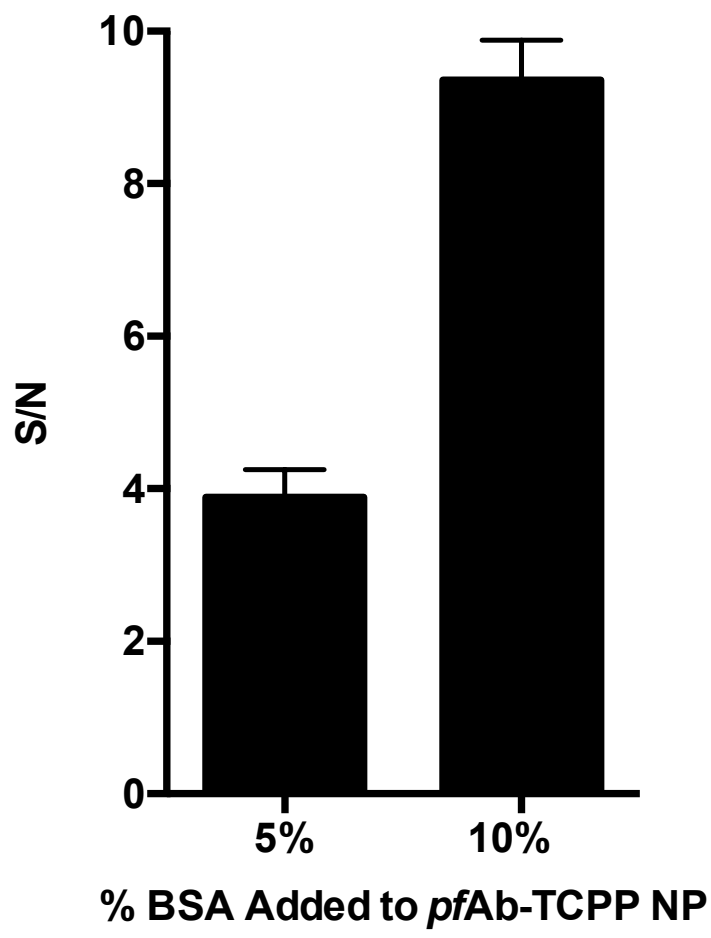

Figure S-11. Blocking of pfAb-TCPP NPs with BSA. Increasing BSA concentration increases signal to noise of the assay for detection of pfHRPII. 


\section{References}

(1) Ramamurthy, S. Multimetallic and Macromolecular Inorganic Photochemistry, 1999; Vol. 4.

(2) Refractive Index. http://macro.Isu.edu/HowTo/solvents/Refractive Index.htm.

(3) Panda, S.; Mohanty, G. C.; Roy, G.; Sahoo, K. Lat. Am. J. Phys. Educ. 2011, 5. 734-740. 